\title{
Left ventricular ischemia in pre-capillary pulmonary hypertension: a cardiovascular magnetic resonance study
}

\author{
Karthigesh Sree Raman ${ }^{1,2,3,4,5}$, Ranjit Shah ${ }^{1,2,3}$, Michael Stokes $^{6}$, Angela Walls ${ }^{7}$, Richard J. Woodman ${ }^{8}$, \\ Rajiv Ananthakrishna ${ }^{1,2,3}$, Jennifer G. Walker ${ }^{2}$, Susanna Proudman ${ }^{9}$, Peter M. Steele ${ }^{6}$, \\ Carmine G. De Pasquale ${ }^{1,2}$, David S. Celermajer ${ }^{10,11}$, Joseph B. Selvanayagam ${ }^{1,2,3}$
}

${ }^{1}$ College of Medicine and Public Health, Flinders University, Flinders, Australia; ${ }^{2}$ Flinders Medical Centre, Flinders, Australia; ${ }^{3}$ Cardiac Imaging Research, South Australian Health \& Medical Research Institute, Australia; ${ }^{4}$ Whangarei Hospital, Northland District Health Board, Whangarei, New Zealand; ${ }^{5}$ Department of Medicine (Northland Campus), Faculty of Medicine and Health Sciences, University of Auckland, Auckland, New Zealand; ${ }^{6}$ Department of Cardiology, Royal Adelaide Hospital, Adelaide, South Australia, Australia; ${ }^{7}$ Clinical Research and Imaging Centre, South Australian Health \& Medical Research Institute, Adelaide, South Australia, Australia; ${ }^{8}$ Flinders Centre of Epidemiology and Biostatistics, College of Medicine and Public Health, Flinders University, Flinders, Australia; ${ }^{9}$ Discipline of Medicine, University of Adelaide, Adelaide, Australia; ${ }^{10}$ Sydney Medical School, University of Sydney and Royal Prince Alfred Hospital, Sydney, Australia; ${ }^{11}$ Department of Cardiology, Royal Prince Alfred Hospital, Sydney, Australia

Contributions: (I) Conception and design: DS Celermajer, JB Selvanayagam; (II) Administrative support: K Sree Raman, A Walls; (III) Provision of study materials or patients: R Shah, M Stokes, S Proudman, JG Walker, R Ananthakrishna, PM Steele; (IV) Collection and assembly of data: K Sree Raman; (V) Data analysis and interpretation: R Woodman, K Sree Raman; (VI) Manuscript writing: All authors; (VII) Final approval of manuscript: All authors.

Correspondence to: Professor Joseph B. Selvanayagam, DPhil. Department of Cardiovascular Medicine, Flinders Medical Centre, Adelaide, South Australia 5042, Australia. Email: Joseph.Selvanayagam@flinders.edu.au.

Background: Prognosis in pulmonary arterial hypertension (PAH) is largely dependent on right ventricular (RV) function. However, recent studies have suggested the presence of left ventricular (LV) dysfunction in $\mathrm{PAH}$ patients. The potential role of LV ischemia, as a contributor to progressive LV dysfunction, has not been systematically studied in PAH. We aim to assess the presence and extent of LV myocardial ischemia in patients with known $\mathrm{PH}$ and without obstructive coronary artery disease (CAD), using oxygen-sensitive (OS) cardiovascular magnetic resonance (CMR) and stress/rest CMR T1 mapping.

Methods: We prospectively recruited 28 patients with right heart catheter-proven $\mathrm{PH}$ and no significant CAD, 8 patients with known CAD and 11 normal age-matched controls (NC). OS-CMR images were acquired using a T2* sequence and T1 maps were acquired using Shortened Modified Look-Locker Inversion recovery (ShMOLLI) at rest and adenosine-induced stress vasodilatation; $\triangle \mathrm{OS}-\mathrm{CMR}$ signal intensity (SI) index (stress/rest SI) and $\Delta \mathrm{T} 1$ reactivity (stress-rest/rest T1 mapping) were calculated.

Results: Global LV $\Delta$ OS SI index was significantly lower in $\mathrm{PH}$ patients compared with controls $(11.1 \% \pm 6.7 \%$ vs. $20.5 \% \pm 10.5 \%, \mathrm{P}=0.016)$, as was $\Delta \mathrm{T} 1$ reactivity $(5.2 \% \pm 4.5 \%$ vs. $8.0 \% \pm 2.9 \%, \mathrm{P}=0.047)$. The ischemic segments of CAD patients had comparable $\triangle$ OS SI $(10.3 \% \pm 6.4 \%$ vs. $11.1 \% \pm 6.7 \%, \mathrm{P}=0.773)$ to $\mathrm{PH}$ patients, but lower $\Delta \mathrm{T} 1$ reactivity $(1.1 \% \pm 4.2 \%$ vs. $5.2 \% \pm 4.5 \%, \mathrm{P}=0.036)$.

Conclusions: Decreased OS-CMR SI and T1 reactivity signify the presence of impaired myocardial oxygenation and vasodilatory response in PH patients. Given their unobstructed epicardial coronary arteries, this is likely secondary to coronary microvascular dysfunction (CMD).

Keywords: Pulmonary hypertension; cardiac magnetic resonance (CMR); coronary microvascular dysfunction (CMD); oxygen-sensitive cardiac magnetic resonance; stress/rest T1 mapping

Submitted Aug 07, 2020. Accepted for publication Sep 24, 2020.

doi: $10.21037 /$ cdt-20-698

View this article at: http://dx.doi.org/10.21037/cdt-20-698 


\section{Introduction}

Pulmonary arterial hypertension (PAH) is a serious and progressive disorder, in which the prognosis is largely dependent on right ventricular (RV) function (1). Recent studies, however, have suggested the presence of left ventricular (LV) dysfunction in PAH patients. Studies from explanted hearts of PAH patients, for example, have demonstrated atrophy and severe contractility impairment of the LV cardiomyocyte (2). The potential contribution of $\mathrm{LV}$ ischemia, to progressive $\mathrm{LV}$ dysfunction has not been systematically studied in PAH.

Oxygen-sensitive (OS) cardiovascular magnetic resonance (CMR), also known as blood oxygen level-dependent (BOLD) CMR imaging, enables the in vivo assessment of myocardial oxygenation at the tissue level. The utility of this technique is underpinned by the natural paramagnetic properties of hemoglobin (3). Following vasodilator stress, healthy vessels dilate sufficiently to increase myocardial oxygenation creating a BOLD-effect, leading to signal intensity (SI) changes in OS-CMR sequence. In segments subtended by healthy vessels there is relative reduction deoxyhemoglobin concentration, leading to a rise in SI. Conversely a mismatch in oxygen supply and demand causes to a relative accumulation of deoxyhemoglobin which would blunt the SI changes. The OS-CMR sequence has been validated by direct measurement of myocardial oxygenation in various conditions involving the left ventricle (LV) (4). In addition to OS-CMR, CMR stress/rest T1 mapping has emerged as a novel promising technique to distinguish normal, ischemic and infarcted myocardium in patients with coronary artery disease (CAD) (5). This technique measures changes in T1-values between native (rest) T1 mapping and vasodilator induced native (stress) T1 mapping. CMR T1 mapping is highly sensitive to changes in myocardial water content, including myocardial blood volume (MBV) $(6,7)$. Vasodilator stress induction causes coronary vasodilatation which leads to changes to myocardial water (6) and MBV $(8,9)$; hence altering T1-values which will enable assessment of microvascular and MBV changes during ischemia (10). Stress/rest T1 mapping is validated to distinguish between epicardial and microvascular CAD (11-14).

While epicardial CAD is the most common cause of myocardial ischemia, studies have demonstrated that coronary microvascular dysfunction (CMD) leads to reduced quality of life and carries an adverse longterm prognosis $(15,16)$. To date, no study has used these emerging CMR techniques to advance the understanding of myocardial ischemia and/or oxygenation in the left ventricle of pre-capillary $\mathrm{PH}$ patients. This could potentially provide novel mechanistic insights into the pathophysiology of the complex PH clinical syndrome and subsequently lead to potential new treatment pathways. Hence, the present study sought to assess the presence and extent of LV myocardial ischemia in patients with known pre-capillary $\mathrm{PH}$ and absent epicardial CAD, using OS-CMR and stress/rest T1 mapping. We present the following article in accordance with the STROBE reporting checklist (available at http:// dx.doi.org/10.21037/cdt-20-698).

\section{Methods}

\section{Study population}

Patients attending $\mathrm{PH}$ clinics at two South Australian public hospitals were invited to participate in this study. The inclusion criteria were right heart catheter-proven precapillary $\mathrm{PH}$ [defined as mean pulmonary artery pressure $(\mathrm{mPAP}) \geq 25 \mathrm{mmHg}$ and pulmonary artery wedge pressure $(\mathrm{PAWP})<15 \mathrm{mmHg}$. Exclusion criteria included severe RV dysfunction on echocardiography (determined by tricuspid annular plane systolic excursion), echocardiographic $\mathrm{LV}$ ejection fraction $<50 \%$ and/or CAD (defined as $>70 \%$ luminal stenosis in an epicardial coronary artery at angiography or prior myocardial infarction) as well as contraindications to CMR and/or adenosine (second or third-degree heart block, obstructive pulmonary disease or dipyridamole use). Eight patients with known CAD (CAD controls) on coronary angiogram were recruited into the study in order to characterize the OS-CMR and T1 reactivity values in their ischemic myocardial segments for comparison with the values in the myocardium of patients with PH. Eleven healthy volunteers (normal controls) with no known cardiac or respiratory disease or symptoms and no cardiac risk factors, including hypertension, smoking and diabetes, were invited to participate as controls. The study was conducted in accordance with the Declaration of Helsinki (as revised in 2013). This study was approved by the Southern Adelaide Clinical Human Research Ethics Committee (HREC/15/SAC/397), and all participants provided written informed consent to participate in the study.

\section{CMR protocol}

The participants were scanned using a 3 Tesla clinical MR 


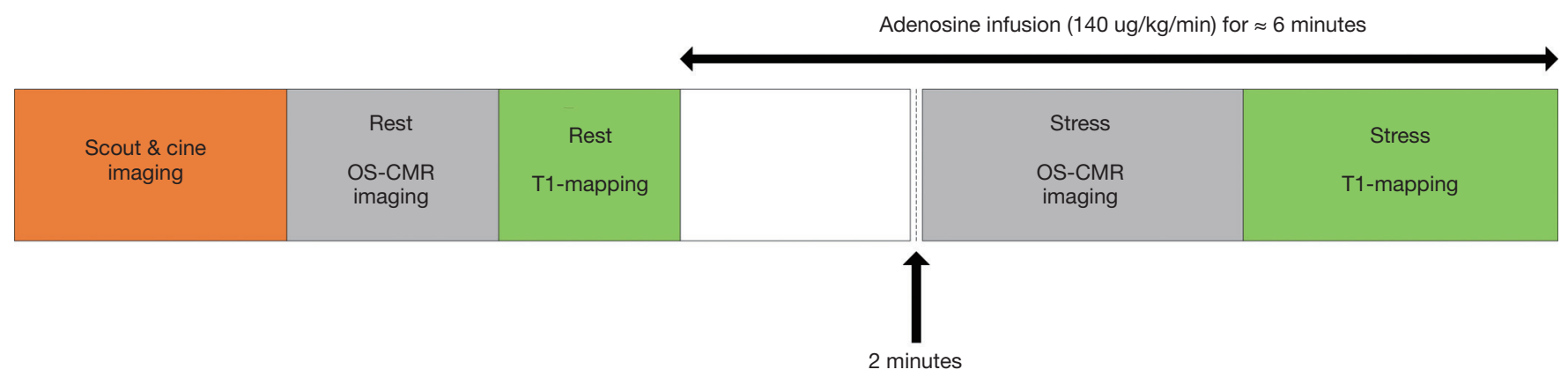

Figure $1 \mathrm{CMR}$ imaging protocol. OS-CMR and rest/stress CMR imaging protocol for PH, CAD and control group. OS-CMR, oxygensensitive cardiovascular magnetic resonance; $\mathrm{CAD}$, coronary artery disease.

scanner (Siemens, 3T MAGNETOM Skyra, 18 channel torso phased array coil in conjunction with a spinal coil posteriorly). The participants were asked to refrain from caffeine 24 hours before the scan. The cine images were acquired in vertical and horizontal long-axis, and ten shortaxis images covering the entire right and left ventricles, using a retrospective ECG gating steady-state free precession (SSFP) sequence [repetition time (TR) $3 \mathrm{~ms}$, echo time (TE) $1.5 \mathrm{~ms}$, flip angle $55^{\circ}$. For OS imaging, a single mid-ventricular slice was acquired at mid-diastole using a T2-prepared ECG-gated SSFP sequence (TR $256 \mathrm{~ms}$, TE $1.21 \mathrm{~ms}$, T2 preparation time $40 \mathrm{~ms}$, matrix $168 \times 192$, field of view $340 \mathrm{~mm} \times 340 \mathrm{~mm}$, slice thickness $6 \mathrm{~mm}$, flip angle $44^{\circ}$ ) (Figure 1). Four OS-CMR images were acquired at rest during a single breath-hold over six heartbeats. At stress, four OS-CMR images identical to the ones acquired at rest were acquired at peak adenosine stress $(140 \mu \mathrm{g} / \mathrm{kg}$ per minute) starting at 120 seconds after initiation for at least 5 minutes (Figure 1). T1 mapping was acquired using Shortened Modified Look-Locker Inversion recovery (ShMOLLI) in three short-axis slice positions (basal, mid-ventricular and apical) as previously described (Figure 1) (5). The resting T1 maps were acquired after the resting OS-CMR images. The mid-ventricular slice location was matched to the mid-ventricular resting OSCMR images, and the basal slice was carefully selected to avoid the LV outflow tract. Stress T1 maps were acquired after the stress OS-CMR images (starting 5 mins after the commencement of the adenosine infusion) in 3 short-axis slices matching the resting T1 maps. Stress heart rate and blood pressure were obtained once every minute during the adenosine infusion. Patients were monitored by ECG, sphygmomanometry and pulse oximetry throughout the study.

\section{CMR analysis}

CMR analysis was performed using $\mathrm{CVI}^{42}$ (Circle CVI, Calgary, Canada). The left and RV function were analyzed quantitatively from the short-axis SSFP images. Standard functional parameters were calculated including $\mathrm{LV}$ ejection fraction (LVEF), RV ejection fraction (RVEF), LV cardiac index (CI), and body surface area indexed end-diastolic (EDVi), end-systolic (ESVi) volumes for LV/RV and LV indexed myocardial mass.

OS-CMR assessment of the LV was performed as previously described (17). In brief, the LV epicardium and endocardium were manually traced and corrected for cardiac motion. The LV myocardium was sub-divided into 6 equiangular segments based on a standard American Heart Association segmentation of the mid-ventricular slice (Figure 2) (18). Mean myocardial SI within each segment was obtained by averaging signal measurements from images acquired both at rest and stress and corrected for heart rate using previously published techniques (19). The measured SI corrections for heart rate were made using the following equation (17):

$$
\mathrm{S}=\mathrm{S}_{0} /\left[1-\beta \mathrm{e}\left(-\mathrm{T}_{\mathrm{R}} / \mathrm{T} 1\right)\right]
$$

An empirical value of $T 1=1,220 \mathrm{~ms}$ and $\beta=0.59$ from previously described work was used for this sequence (17). $\mathrm{S}$ is the corrected SI and $\mathrm{S}_{0}$ is the measured SI. TR is the image dependent time between acquisitions of sections of $k$-space, governed by the heart rate (17). The relative SI change was calculated as $\Delta \mathrm{SI}(\%)=(\mathrm{SI}$ stress $-\mathrm{SI}$ rest $) / \mathrm{SI}$ rest $\times 100$. The mean global $\mathrm{LV} \triangle \mathrm{OS}-\mathrm{CMR}$ SI is derived from the mean $\triangle \mathrm{OS}-\mathrm{CMR}$ SI of all 6 equiangular segments. The mean LV septal $\triangle \mathrm{OS}-\mathrm{CMR}$ SI was derived from the mean $\triangle$ OS-CMR SI of the antero- and inferoseptal LV segments and the mean LV free-wall $\triangle \mathrm{OS}-\mathrm{CMR}$ SI was 


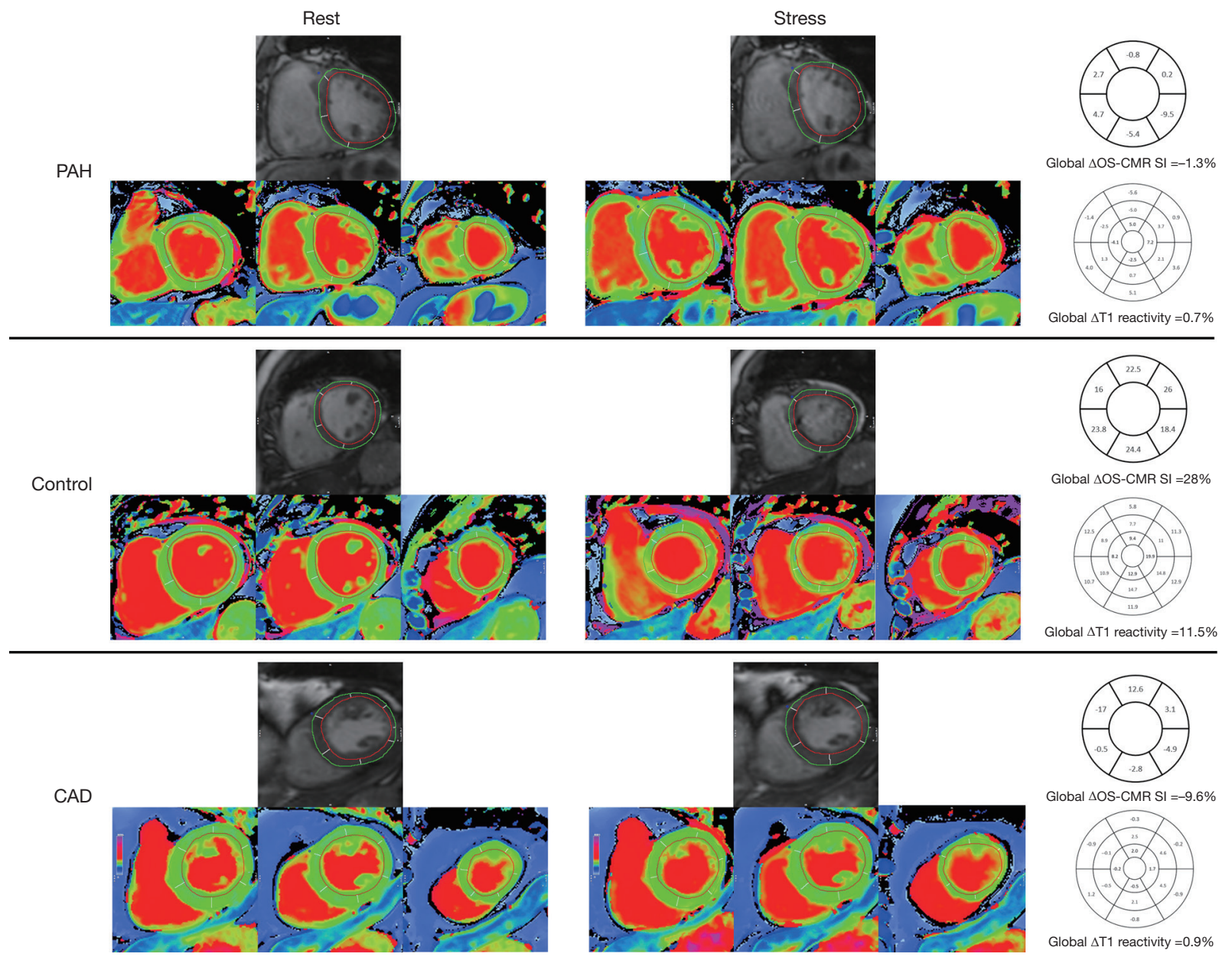

Figure 2 Representative cases in a PH, control and CAD patient. OS-CMR and Rest/Stress T1 mapping protocol whereby a resting midventricular OS-CMR is first acquired followed by the resting T1 mapping at basal, mid-ventricular (matched to OS-CMR image slice location) and apical. Following vasodilator stress, matching mid ventricular slice stress OS-CMR images and stress T1 mapping images matching the resting three short-axis slice positions were then acquired. OS-CMR, oxygen-sensitive cardiovascular magnetic resonance; CAD, coronary artery disease.

derived from the mean $\triangle \mathrm{OS}-\mathrm{CMR}$ SI of the antero- and inferolateral LV segments.

Myocardial T1 analysis was performed on the T1 maps acquired during rest and stress. The endocardial and epicardial contours were manually traced for all the 3 shortaxis T1 map images (basal, mid-ventricular and apical) and were divided into 16 segments according to the American Heart Association 17-segment model (18). The mean myocardial T1 within each segment was obtained, both at rest and stress. The $\mathrm{T} 1$ reactivity $(\Delta \mathrm{T} 1)$ was then calculated from the $\mathrm{T} 1$-values at rest $\left(\mathrm{T} 1_{\text {Rest }}\right)$ and during adenosine stress $\left(\mathrm{T} 1_{\text {Stress }}\right)$ as (Figure 2$)$ :

$$
\Delta \mathrm{T} 1=\left(\mathrm{T} 1_{\text {Stress }}-\mathrm{T} 1_{\text {Rest }}\right) / \mathrm{T} 1_{\text {Rest }} \times 100 \%
$$

\section{Statistical analysis}

All analyses were performed using the Stata statistical software version 15.1 (StataCorp., USA). Categorical data are described using frequencies and percentages and continuous data using mean and standard deviation. Differences between groups in mean resting $\mathrm{T} 1$, the mean changes in $\mathrm{T} 1$ and the mean changes in myocardial oxygenation response were assessed using independent $t$-tests. Group comparisons were made using ANOVA with post-hoc Dunnett's test used for multiple group corrections. Changes in these same outcomes within patient groups were assessed using paired $t$-tests. The overall association between OS-CMR and T1 reactivity was assessed using 
Table 1 Patient demographics and baseline clinical data

\begin{tabular}{|c|c|c|c|c|}
\hline Variables & $\mathrm{PH}(\mathrm{n}=28)$ & CAD $(n=8)$ & Control $(n=11)$ & $P$ value \\
\hline Females sex, $\mathrm{n}(\%)$ & $19(68)$ & $4(50)$ & $4(36 \%)$ & $0.291^{\ddagger}$ \\
\hline \multicolumn{5}{|l|}{ Comorbidities } \\
\hline Hypertension, n (\%) & $15(54)$ & $7(87.5)$ & 0 & \\
\hline Dyslipidaemia, n (\%) & $8(29)$ & $2(25)$ & 0 & \\
\hline Chronic obstructive airways disease, $\mathrm{n}(\%)$ & $9(32)$ & 0 & 0 & \\
\hline Obstructive sleep apnoea, n (\%) & $5(18)$ & 0 & 0 & \\
\hline Atrial fibrillation, $\mathrm{n}(\%)$ & $6(21)$ & 0 & 0 & \\
\hline Pulmonary artery wedge pressure $(\mathrm{mmHg})$ & $12 \pm 3$ & $\mathrm{~N} / \mathrm{A}$ & $\mathrm{N} / \mathrm{A}$ & \\
\hline Mean right atrial pressures $(\mathrm{mmHg})$ & $11 \pm 4$ & $\mathrm{~N} / \mathrm{A}$ & $\mathrm{N} / \mathrm{A}$ & \\
\hline Cardiac index $\left(\mathrm{L} / \mathrm{min} / \mathrm{m}^{2}\right)$ & $2.8 \pm 0.7$ & N/A & N/A & \\
\hline Pulmonary vascular resistance index (Woods unit $\mathrm{m}^{2}$ ) & $8.3 \pm 4.7$ & N/A & N/A & \\
\hline \multicolumn{5}{|l|}{ Medication } \\
\hline Aspirin, n (\%) & $4(14)$ & $7(88)$ & 0 & \\
\hline Beta-blockers, n (\%) & $9(32)$ & $5(63)$ & 0 & \\
\hline Soluble guanylate cyclase, $n(\%)$ & $2(7)$ & 0 & 0 & \\
\hline Combination therapy ${ }^{*}, \mathrm{n}(\%)$ & $14(50)$ & 0 & 0 & \\
\hline
\end{tabular}

*, combination of either endothelin receptor blocker, PDE5 inhibitor or soluble guanylate cyclase; ${ }^{\ddagger}$, Chi-square test; ${ }^{\S}$, P value from oneway ANOVA. PH, pulmonary hypertension; CAD, coronary artery disease; SD, standard deviation; ACEi, angiotensin-converting enzyme inhibitors; ARB, angiotensin II receptor blockers; PDE5, phosphodiesterase type 5 inhibitor.

a mixed effects model to account for non-independence of observations due to multiple measures from different segments. We included a random intercept for the subject as well as a fixed effect for the segment. Separate associations between OS-CMR and T1 reactivity for each segment were assessed using the Pearson $\mathrm{r}$ correlation coefficient. A 2 -sided type 1 error rate of alpha $=0.05$ was used for assessing statistical significance.

\section{Results}

\section{Participants characteristics}

The subject characteristics are summarized in Tables 1,2. Among the $\mathrm{PH}$ patients, 11 (39\%) had idiopathic pulmonary artery hypertension (iPAH), 13 (46\%) patients had systemic sclerosis-associated PAH (SSc-PAH), and 4 (14\%) patients had chronic thromboembolic PH (CTEPH). Average 
Table 2 CMR Ventricular Function

\begin{tabular}{|c|c|c|c|c|}
\hline Variables & $\mathrm{PH}(\mathrm{n}=28)$ & $\mathrm{CAD}(\mathrm{n}=8)$ & Control $(n=11)$ & P value \\
\hline LV EDVi $\left(\mathrm{mL} / \mathrm{m}^{2}\right)$, mean $\pm \mathrm{SD}$ & $72 \pm 15$ & $88 \pm 38$ & $64 \pm 11$ & 0.194 \\
\hline LV ESVi $\left(\mathrm{mL} / \mathrm{m}^{2}\right)$, mean \pm SD & $24 \pm 10$ & $39 \pm 28$ & $20 \pm 4$ & 0.131 \\
\hline LV ED mass index $\left(\mathrm{g} / \mathrm{m}^{2}\right)$, mean $\pm \mathrm{SD}$ & $53 \pm 9$ & $58 \pm 20$ & $51 \pm 9$ & 0.589 \\
\hline $\mathrm{LV} C \mathrm{Cl}\left(\mathrm{L} / \mathrm{min} / \mathrm{m}^{2}\right)$, mean $\pm \mathrm{SD}$ & $3.2 \pm 0.6$ & $3.0 \pm 0.7$ & $2.8 \pm 0.3$ & 0.154 \\
\hline RVEF (\%), mean \pm SD & $60 \pm 9$ & $74 \pm 5$ & $64 \pm 8$ & $<0.001$ \\
\hline RV EDVi $\left(\mathrm{mL} / \mathrm{m}^{2}\right)$, mean $\pm \mathrm{SD}$ & $66 \pm 17$ & $66 \pm 20$ & $63 \pm 13$ & 0.878 \\
\hline RV ESVi $\left(m L / m^{2}\right)$, mean \pm SD & $27 \pm 12$ & $17 \pm 7$ & $23 \pm 8$ & 0.020 \\
\hline
\end{tabular}

§, P value from one-way ANOVA. CMR, cardiac magnetic resonance; LV, left ventricle; RV, right ventricle; EF, ejection fraction; EDVi, enddiastolic volume index; ESVi, end-systolic volume index; ED, end-diastolic; SV, stroke volume; Cl, cardiac index; SD, standard deviation.

mPAP was $34 \pm 7 \mathrm{mmHg}$ and the average mean PAWP was $12 \pm 3 \mathrm{mmHg}$. The mean interval between right heart catheter with coronary angiogram and the CMR research study was 2 years. The mean 6-minute walk distance was $392 \pm 114$ meters. On echocardiography, 9 (36\%) patients had RV dilatation with an echocardiography-estimated resting systolic pulmonary artery pressure of $52 \pm 25 \mathrm{mmHg}$. All PH patients were treated with pulmonary vasodilators such as bosentan, macitentan, sildenafil, riociguat with $14(50 \%)$ on combination therapy. The $\mathrm{PH}$ patients were stable and continued their pulmonary vasodilator therapy during the CMR research scan.

\section{CMR characteristics}

Table 2 shows a comparison of CMR variables between $\mathrm{PH}, \mathrm{CAD}$ and controls. The only statistically significant difference in CMR volumetric and functional indices between $\mathrm{PH}, \mathrm{CAD}$ and controls was the RVEF $(\mathrm{P}<0.001)$ and RV ESVi $(\mathrm{P}=0.020)$.

\section{$L V$ myocardial oxygenation response (OS-CMR)}

All 28 patients completed the OS-CMR study protocol. The mean global LV $\triangle$ OS-CMR SI change was significantly lower in the $\mathrm{PH}$ group compared to the controls ( $11.1 \% \pm 6.7 \%$ vs. $20.5 \% \pm 10.5 \%, \mathrm{P}=0.016)$. In contrast, the $\triangle$ OS-CMR SI changes in the myocardium of the $\mathrm{PH}$ patients were comparable to the ischemic segments of CAD patients $(11.1 \% \pm 6.7 \%$ vs. $10.3 \% \pm 6.4 \%, \mathrm{P}=0.773)$ (central illustration). As there is interventricular 'septal bowing' (D shaping) in $\mathrm{PH}$ we also compared the $\triangle \mathrm{OS}-\mathrm{CMR}$ SI in various segments of the $\mathrm{LV}$. We found in $\mathrm{PH}$, the mean global LV $\triangle$ OS-CMR SI was comparable to mean LV septal $\triangle$ OS-CMR SI $(11.1 \% \pm 6.7 \%$ vs. $12.3 \% \pm 7.6 \%, \mathrm{P}=0.523)$ and mean LV free-wall $\triangle$ OS-CMR SI $(11.1 \% \pm 6.7 \%$ vs. $10.3 \% \pm 8.2 \%, \mathrm{P}=0.710)$. In addition, there was comparable mean $\triangle$ OS-CMR SI changes between LV septal and LV free-wall $(12.3 \% \pm 7.6 \%$ vs. $10.3 \% \pm 8.2 \%, \mathrm{P}=0.352)$ in the $\mathrm{PH}$ group.

\section{Myocardial stress/rest T1 reactivity}

Of the total of $28 \mathrm{PH}$ patients who underwent the Stress/ Rest T1 CMR protocol, 23 (82\%) patients had good quality analyzable T1 maps. Stress/Rest T1 maps were excluded due to the presence of LV outflow tract on the basal T1 map slices at stress in $3(11 \%)$ patients and the remaining 2 (7\%) patients had poor T1 maps due to cardiac or respiratory motion. Resting T1-values for the control were within previously published ranges: $1,153 \pm 33$ (14). Compared to controls, patients with $\mathrm{PH}$ had higher resting T1-values: $1,247 \pm 74, \mathrm{P}<0.0001$. During vasodilator stress, the T1-values increased significantly in both $\mathrm{PH}$ patients (from $1,247 \pm 74$ to $1,311 \pm 83, \mathrm{P}<0.0001$ ) and controls (from $1,153 \pm 33$ to $1,245 \pm 38, \mathrm{P}<0.0001$ ). However, the global $\Delta \mathrm{T} 1$ reactivity was significantly blunted in the $\mathrm{PH}$ patients compared to controls $(5.2 \% \pm 4.5 \%$ vs. $8.0 \% \pm 2.9 \%$, 

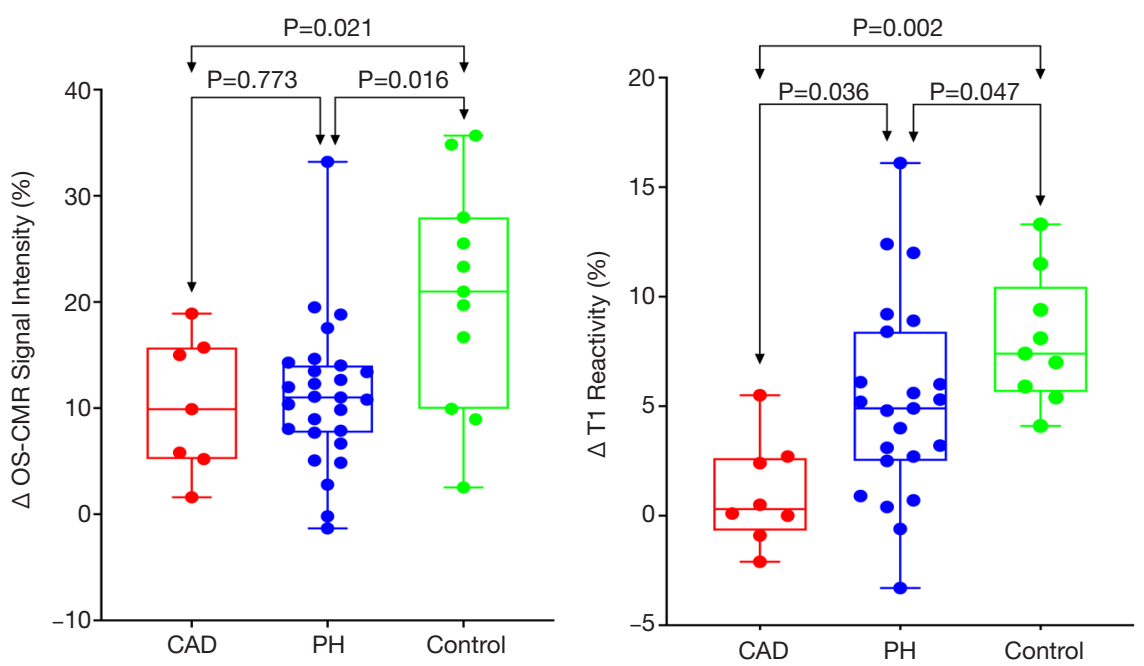

Figure 3 Distribution of vasodilator stress response of OS-CMR and T1 Reactivity in coronary artery disease (CAD), pulmonary hypertension (PH) and control. The $\triangle \mathrm{OS}-\mathrm{CMR}$ SI in $\mathrm{PH}$ was comparable to CAD patients but significantly lower compared to control group. Meanwhile there is a significant difference of $\triangle \mathrm{T} 1$ reactivity in $\mathrm{PH}$ compared to $\mathrm{CAD}$ and control group.

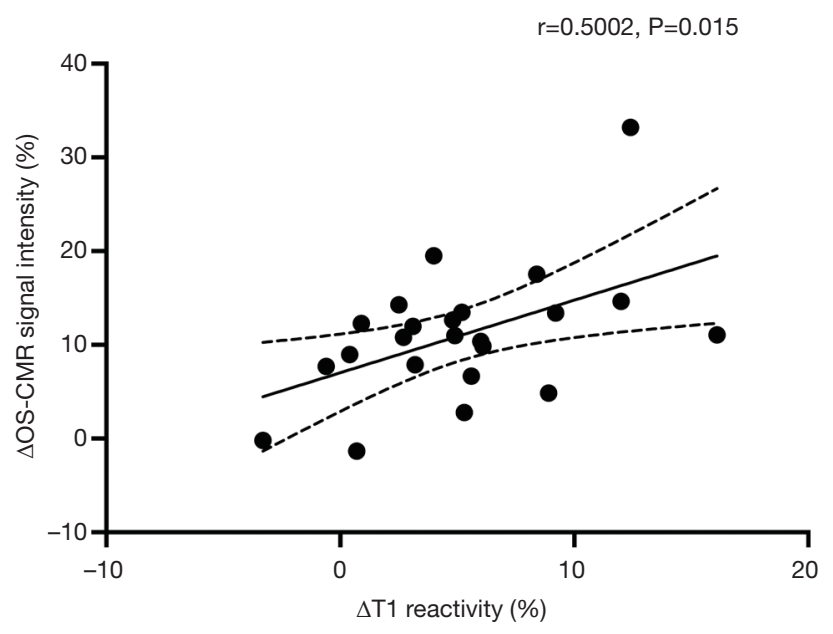

Figure 4 Patient correlation between OS-CMR and T1 Reactivity. Among $\mathrm{PH}$ patients, there is moderate correlation between global $\triangle \mathrm{OS}-\mathrm{CMR}$ and global $\Delta \mathrm{T} 1$ reactivity. OS-CMR, oxygen-sensitive cardiovascular magnetic resonance.

$\mathrm{P}=0.047)$. The ischemic segments of CAD patients had comparable resting T1 value to controls $(1,197 \pm 78 \mathrm{vs}$. $1,153 \pm 33, \mathrm{P}=0.170)$ and $\mathrm{PH}$ patients $(1,197 \pm 78$ vs. $1,247 \pm 74$, $\mathrm{P}=0.138)$. However, the $\Delta \mathrm{T} 1$ reactivity in the ischemic segments of CAD was $1.1 \% \pm 4.2 \%$ and this is significantly lower when compared to the global $\Delta \mathrm{T} 1$ reactivity in $\mathrm{PH}$ patients $(5.2 \% \pm 4.5 \%, \mathrm{P}=0.036)$ and controls $(8.0 \% \pm 2.9 \%, \mathrm{P}=0.002)$ (Figure 3). In $\mathrm{PH}$, the global $\mathrm{LV}$
$\Delta \mathrm{T} 1$ reactivity was comparable to mean $\mathrm{LV}$ septal $\Delta \mathrm{T} 1$ reactivity $(5.2 \% \pm 4.5 \%$ vs. $4.4 \% \pm 3.7 \%, \mathrm{P}=0.537)$ and mean $\mathrm{LV}$ free-wall $\Delta \mathrm{T} 1$ reactivity $(5.2 \% \pm 4.5 \%$ vs. $5.8 \% \pm 4.9 \%$, $\mathrm{P}=0.625)$. In addition, there was no significant difference between mean $\Delta \mathrm{T} 1$ reactivity of $\mathrm{LV}$ septal and $\mathrm{LV}$ free-wall $(4.4 \% \pm 3.7 \%$ vs. $5.8 \% \pm 4.9 \%, \mathrm{P}=0.267)$.

\section{Comparing $L V$ myocardial ischemia in iPAH and SSc-PAH}

No significantly different changes in global $\triangle \mathrm{OS}-\mathrm{CMR}$ SI or $\Delta \mathrm{T} 1$ reactivity were seen between iPAH and SScPAH patients. The global LV $\triangle$ OS-CMR SI in the iPAH $(\mathrm{n}=11)$ and SSc-PAH $(\mathrm{n}=13)$ patients was $9.7 \% \pm 5.1 \%$ vs. $11.5 \% \pm 7.9 \%, \mathrm{P}=0.516$. The global $\Delta \mathrm{T} 1$ reactivity between the $\mathrm{iPAH}(\mathrm{n}=9)$ and SSc-PAH $(\mathrm{n}=13)$ patients was $6.5 \% \pm 5.3 \%$ vs. $4.3 \% \pm 4.0 \%, \mathrm{P}=0.319$. The CTEPH patients were not compared due to low patient numbers.

\section{Correlations between myocardial oxygenation, $\triangle T 1$ reactivity and CMR volumetric/functional and bemodynamic indices in $\mathrm{PH}$ patients}

When the changes in OS-CMR and T1 were averaged, there was a moderate correlation between global $\triangle \mathrm{OS}$ CMR SI and $\Delta \mathrm{T} 1$ reactivity $(\mathrm{r}=0.50, \mathrm{P}=0.015)$ (Figure 4). However, a stratified segmental analysis between a midventricular $\triangle \mathrm{OS}-\mathrm{CMR}$ SI and mid-ventricular $\Delta \mathrm{T} 1$ reactivity showed mostly weak associations with Pearson $\mathrm{r}$ correlation coefficients for the 6 segments of $0.46(\mathrm{P}=0.03)$, 


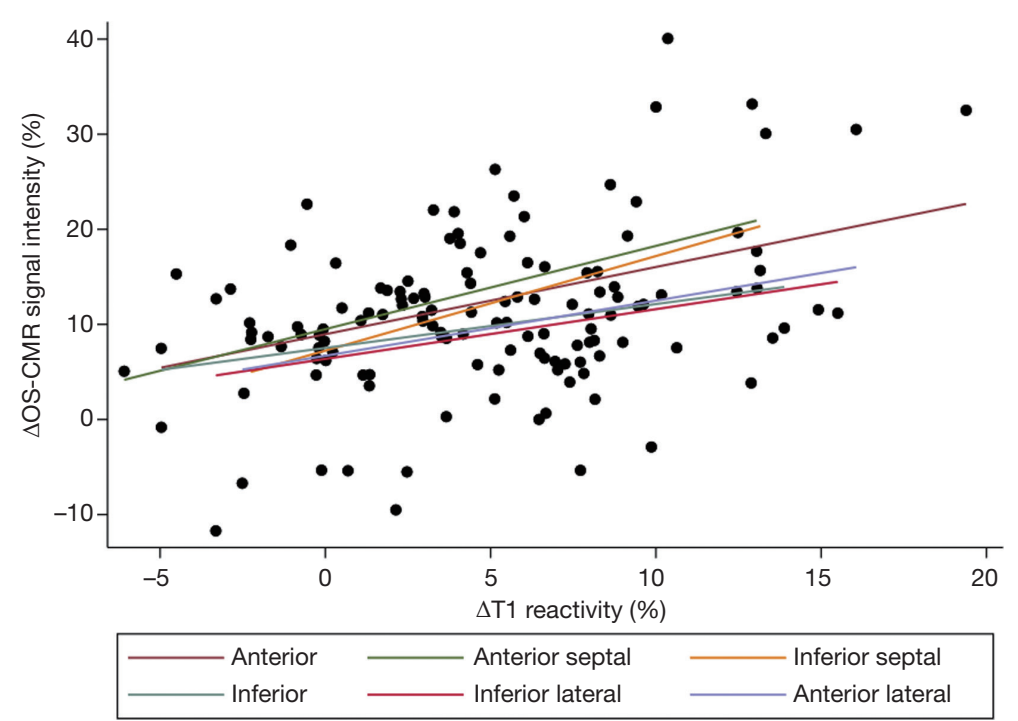

Figure 5 Segmental correlation between OS-CMR and T1 Reactivity. On mid ventricular segmental comparison among PH patients, there is weaker correlation between $\triangle \mathrm{OS}-\mathrm{CMR}$ and $\triangle \mathrm{T} 1$ reactivity. OS-CMR, oxygen-sensitive cardiovascular magnetic resonance.

$0.50(\mathrm{P}=0.02), 0.54(\mathrm{P}=0.009), 0.31(\mathrm{P}=0.16), 0.28(\mathrm{P}=0.20)$ and $0.35(\mathrm{P}=0.11)$ (Figure 5). In addition, there was no overall association between mid-ventricular $\triangle$ OS-CMR SI and mid-ventricular $\Delta \mathrm{T} 1$ reactivity when assessed using the mixed effects model ( $\beta=-0.05,95 \% \mathrm{CI},-0.32,0.22 ; \mathrm{P}=0.71$ ). These findings imply that while a blunted global $\Delta$ OSCMR SI and $\Delta \mathrm{T} 1$ reactivity suggest presence of abnormal myocardial oxygenation and vasodilatory response, there is poor association between blunted myocardial deoxygenation and $\Delta \mathrm{T} 1$ reactivity. Compared to $\mathrm{CMR}$ volumetric/functional indices $\triangle$ OS-CMR SI had moderate correlation to CMR RV EDVi ( $\mathrm{r}=-0.47, \mathrm{P}=0.016)$ and CMR LVEF ( $\mathrm{r}=0.41, \mathrm{P}=0.038) . \Delta \mathrm{T} 1$ reactivity had strong correlation to CMR RV EDVi $(\mathrm{r}=-0.73, \mathrm{P}<0.001)$ and a moderate correlation to CMR LVEF ( $\mathrm{r}=0.48, \mathrm{P}=0.023)$. These findings suggest that increased CMR RV EDVi and decreased CMR LVEF are associated with blunted $\triangle \mathrm{OS}$ CMR SI and $\Delta \mathrm{T} 1$ reactivity (Figure 6 ). There was no significant correlation between myocardial oxygenation and $\Delta \mathrm{T} 1$ reactivity to $\mathrm{RV}$ haemodynamic indices.

\section{Discussion}

In this study, we have demonstrated the presence of blunted myocardial oxygenation and $\mathrm{T} 1$ reactivity in the $\mathrm{LV}$ of $\mathrm{PH}$ patients. These blunted responses to adenosine stress was demonstrated in $\mathrm{PH}$ patients who were stable on pulmonary vasodilator therapy and at an early 'adaptive' stage of $\mathrm{PH}$ with non-obstructive epicardial coronaries. These data strongly suggest the presence of CMD. This provides a novel mechanistic understanding into the pathophysiology of $\mathrm{LV}$ dysfunction in $\mathrm{PH}$, and could subsequently lead to new potential treatment targets, in such patients.

\section{Coronary microvascular disease of the $L V$ in pre-capillary $\mathrm{PH}$}

Several studies have described different pathophysiological causes of LV dysfunction in patients with PAH (20-25). Changes in RV affect the interventricular interaction by leftward septal bowing, which leads to impaired LV diastolic filling $(20,21)$. Other studies have also demonstrated LV diastolic dysfunction $(23,24)$ and reduction in $L V$ free wall mass, in association with RV dysfunction, in PAH (25).

It has been recognized that in PAH, RV myocardial ischemia is a precipitant of adverse remodeling and progressive RV dysfunction (26). The gradual remodeling of small distal pulmonary arteries followed by elevated pulmonary vascular resistance, microvascular ischemia and mitochondrial dysfunction culminates in RV failure and death (26). Although LV myocardial ischemia has been described in congenital heart and left heart disease associated post-capillary pulmonary hypertension, there are limited studies in PAH. In SSc-PAH, the LV myocardial perfusion abnormalities have been attributed to decreased coronary flow reserve (27) and CMD (28) related to 

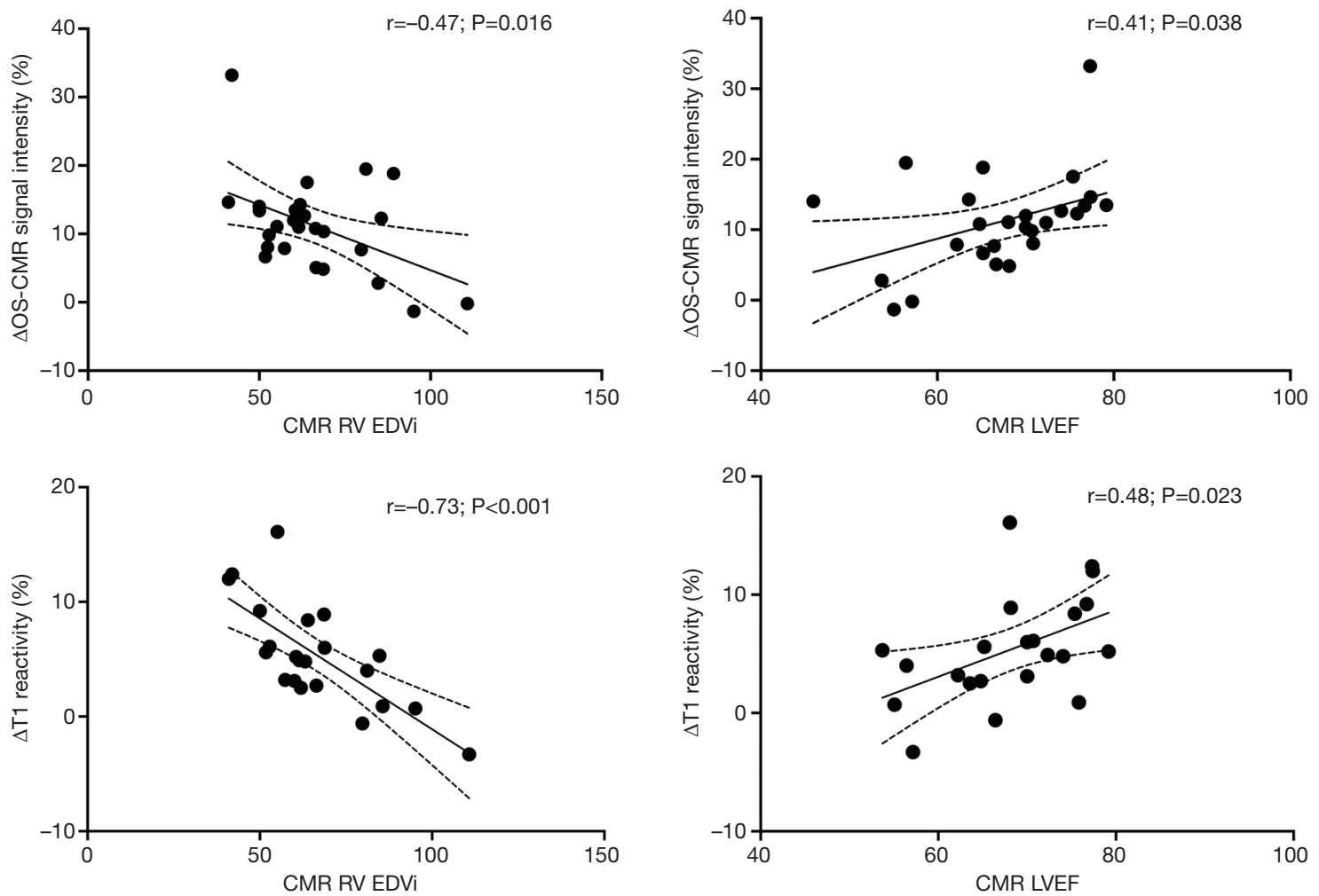

Figure 6 Correlation between OS-CMR, T1 Reactivity and CMR volumetric and functional indices. $\triangle$ OS-CMR had a moderate correlation to CMR right ventricular (RV) end diastolic volume index (EDVi) and CMR left ventricular (LV) ejection fraction (EF). $\triangle \mathrm{T} 1 \mathrm{reactivity}$ had a strong correlation to $\mathrm{CMR}$ right ventricular (RV) end diastolic volume index (EDVi) and a moderate correlation to CMR left ventricular $(\mathrm{LV})$ ejection fraction (EF). OS-CMR, oxygen-sensitive cardiovascular magnetic resonance.

concurrent SSc involvement of the myocardium. Extrinsic compression of the left main coronary arteries (LMCA) due to a dilated pulmonary artery has been elegantly described by Galiè et al. (29). It is increasingly recognized however, that the microvascular damage seen in the pulmonary vasculature of PAH has links to coronary vascular disease. A recent study by Meloche et al. demonstrated that the inflammation and epigenetic readers seen in PAH pulmonary vasculature were also overexpressed in the coronary arteries. The authors suggested that inflammatory and epigenetic readers may trigger coronary vascular remodeling in both the LV and RV leading to CMD and CAD (30). We have now extended these findings by demonstrating that patients with less severe $\mathrm{PH}$, and no obstructive $\mathrm{CAD}$, have a blunted myocardial oxygenation response and stress/rest $\mathrm{T} 1$ response to adenosine stress. These findings are consistent with the presence of $\mathrm{CMD}$ in pre-capillary $\mathrm{PH}$ patients.

\section{Myocardial oxygenation and $T 1$ reactivity in response to vasodilator stress}

Elevated resting (native) T1-values with a significant rise in stress T1-values on vasodilator stress in $\mathrm{PH}$ has been demonstrated in aortic stenosis (11), chronic kidney disease (14), and primary cardiomyopathies $(31,32)$. Native CMR T1 parametric mapping has been used for inflammation/edema imaging and as a proxy for diffuse interstitial fibrosis in the absence of an alternative cause of interstitial expansion (edema, infiltrations/fiber disarray) (33). T1 mapping measures the total water content in the tissue and any changes to intravascular MBV will prolong T1 relaxation time. Vasodilator stress-induced coronary vasodilatation will lead to changes to $\mathrm{MBV}$ which forms the basis of stress/rest T1 mapping. Preliminary studies using stress/rest T1-mapping and CMR first-pass 
perfusion imaging in hypertrophic (31) and dilated (32) cardiomyopathy have demonstrated a correlation between T1 reactivity and myocardial perfusion. While this is encouraging, more in-depth validation of stress/rest T1 mapping is required. In our study, patient tolerability limited the use of CMR first-pass perfusion imaging, however other studies have demonstrated myocardial perfusion abnormalities on CMR first-pass perfusion imaging in PAH (34). Furthermore, elevated myocardial T1-values have been demonstrated in PAH (35), which appears to be consistent with myocardial interstitial space expansion (36). While PAH associated LV underfilling is thought to be the cause of $\mathrm{LV}$ interstitial changes, the exact mechanism is not completely understood $(37,38)$. In addition, there were no differences in the elevated T1-values between iPAH, SSc-PAH or CTEPH $(35,39)$. Therefore, the observed blunted $\Delta \mathrm{T} 1$ reactivity in $\mathrm{PAH}$ occurs as a result altered intravascular MBV during stress secondary to coronary vascular remodeling.

Previous studies have shown that myocardial oxygenation and perfusion during vasodilator stress may be dissociated (40). For example, our group has previously shown that in patients with known CAD, there was a disassociation between OS-CMR SI and quantitative perfusion myocardial blood flow with $50 \%$ of hypoperfused segments demonstrating no evidence of deoxygenation (40). In dilated cardiomyopathy, Dass et al. demonstrated a disassociation between microvascular dysfunction and oxygenation (41). Conversely, oxygen demand may be increased in the absence of perfusion abnormalities as demonstrated in hypertrophic cardiomyopathy gene carriers (42) despite the absence of LV hypertrophy (43). In this study, we have demonstrated a blunted global $\triangle$ OS-CMR SI and global $\Delta \mathrm{T} 1$ reactivity in response to vasodilator stress suggesting the presence of CMD. Furthermore the $\triangle \mathrm{OS}-\mathrm{CMR}$ SI and $\Delta \mathrm{T} 1$ reactivity affects the $\mathrm{LV}$ globally with comparable findings in the LV septal and free wall segments. However, on segmental analysis there is poor correlation between myocardial deoxygenation and changes to intravascular $\mathrm{MBV}$ (i.e., $\triangle \mathrm{T} 1$ reactivity), suggesting that myocardial oxygenation and perfusion may be dissociated in $\mathrm{PH}$ patients also. This interplay between myocardial deoxygenation and changes in intravascular MBV during stress in pre-capillary $\mathrm{PH}$ has not been previously demonstrated.

\section{Study limitations}

Our study only performed a single mid-ventricular slice on OS-CMR imaging rather than the entire ventricle. However, we believe that in $\mathrm{PH}$, the myocardium is affected globally and it is unlikely to affect the results. The OSCMR images were acquired in mid-diastole as opposed to systole. While there are studies that have demonstrated advantages on acquiring OS-CMR images in systole (44), we have chosen mid-diastole acquisition to keep it consistent with T1 mapping acquisition. Moreover, the mid-diastole OS-CMR acquisition was also consistent with our and other studies that have demonstrated microvascular dysfunction in the LV (45-48). This study has a small sample size which could increase the margin of error. A further larger study would help determine the utility of these novel techniques in $\mathrm{PAH}$ and its association with clinical outcomes. Moreover, compared to the PH group, the numbers in the CAD and control groups were relatively small. Nonetheless, both CAD and control groups were agematched to the $\mathrm{PH}$ group, minimizing any significant bias. This is important, especially in T1-values whereby age is known to influence native myocardial T1-values (7). While this was a prospective study, we recruited $\mathrm{PH}$ patients who were stable on pulmonary vasodilator therapy which would have mitigated against the finding of $L V$ ischemia. A larger study would help to determine the clinical and prognostic utility of these novel CMR techniques in $\mathrm{PH}$.

\section{Clinical implication and future directions}

The current study provides insights into the myocardial perfusion and oxygenation abnormalities in patients with non-severe $\mathrm{PH}$. These findings suggest the presence of CMD, which has important clinical implications. Patients with CMD may present with typical effortinduced angina, but also with atypical symptoms such as dyspnea on exertion (49). In PAH, exertional limitation is the dominant symptom, and there are other factors (respiratory mechanics/ventilation, cardiovascular response and psychological/emotional aspects) that can contribute to this (50). Although resting hemodynamic measures such as mPAP and cardiac output correlate with severity of symptoms, there is a substantial interindividual variability that is not explained by hemodynamic severity (51). Although, we have not demonstrated any significant correlation between these novel CMR techniques and conventional prognostic markers of CMR RV EF and hemodynamic indices, OS-CMR and Stress/Rest T1 mapping techniques may provide additional pathophysiological insights in $\mathrm{PH}$ patients with 
ongoing exertional symptoms. In addition, characterizing CMD adds further potential for newer therapies that target myocardial ischemia and oxidative stress, such as ranolazine and trimetazidine that are currently in clinical trials (52).

\section{Conclusions}

Blunted OS-CMR SI and T1 reactivity with stress suggest the presence of CMD in pre-capillary $\mathrm{PH}$ patients without significant epicardial CAD. This provides novel pathophysiological insights and may suggest potential future therapeutic targets, in $\mathrm{PH}$.

\section{Acknowledgments}

Funding: This research is being funded by the National Health and Medical Research Council of Australia and Actelion Pharmaceuticals Australia.

\section{Footnote}

Reporting Checklist: The authors have completed the STROBE reporting checklist. available at http://dx.doi. org/10.21037/cdt-20-698

Data Sharing Statement: Available at http://dx.doi. org/10.21037/cdt-20-698

Conflicts of Interest: All authors have completed the ICMJE uniform disclosure form (available at http://dx.doi. org/10.21037/cdt-20-698). Prof. JBS reports that he serves as a current Editorial Board Member. Dr. SP and Dr. JGW receive grants from Actelion, outside from submitted work. The authors have no other conflicts of interest to declare.

Ethical Statement: The authors are accountable for all aspects of the work in ensuring that questions related to the accuracy or integrity of any part of the work are appropriately investigated and resolved. The study was conducted in accordance with the Declaration of Helsinki (as revised in 2013). The study was approved by Southern Adelaide Clinical Human Research Ethics Committee (HREC/15/SAC/397) and informed consent was taken from all individual participants.

Open Access Statement: This is an Open Access article distributed in accordance with the Creative Commons
Attribution-NonCommercial-NoDerivs 4.0 International License (CC BY-NC-ND 4.0), which permits the noncommercial replication and distribution of the article with the strict proviso that no changes or edits are made and the original work is properly cited (including links to both the formal publication through the relevant DOI and the license). See: https://creativecommons.org/licenses/by-nc-nd/4.0/.

\section{References}

1. Humbert M, Sitbon O, Chaouat A, et al. Survival in patients with idiopathic, familial, and anorexigenassociated pulmonary arterial hypertension in the modern management era. Circulation 2010;122:156-63.

2. Manders E, Bogaard HJ, Handoko ML, et al. Contractile Dysfunction of Left Ventricular Cardiomyocytes in Patients With Pulmonary Arterial Hypertension. J Am Coll Cardiol 2014;64:28-37.

3. Pauling L, Coryell CD. The magnetic properties and structure of hemoglobin, oxyhemoglobin and carbonmonoxyhemoglobin. Proc Natl Acad Sci U S A 1936;22:210-6.

4. Sree Raman K, Nucifora G, Selvanayagam JB. Novel cardiovascular magnetic resonance oxygenation approaches in understanding pathophysiology of cardiac diseases. Clin Exp Pharmacol Physiol 2018;45:475-80.

5. Liu A, Wijesurendra RS, Francis JM, et al. Adenosine Stress and Rest T1 Mapping Can Differentiate Between Ischemic, Infarcted, Remote, and Normal Myocardium Without the Need for Gadolinium Contrast Agents. JACC Cardiovasc Imaging 2016;9:27-36.

6. Piechnik SK, Ferreira VM, Dall'Armellina E, et al. Shortened Modified Look-Locker Inversion recovery (ShMOLLI) for clinical myocardial T1-mapping at 1.5 and $3 \mathrm{~T}$ within a 9 heartbeat breathhold. J Cardiovasc Magn Reson 2010;12:69.

7. Piechnik SK, Ferreira VM, Lewandowski AJ, et al. Normal variation of magnetic resonance $\mathrm{T} 1$ relaxation times in the human population at $1.5 \mathrm{~T}$ using ShMOLLI. J Cardiovase Magn Reson 2013;15:13.

8. McCommis KS, Zhang H, Goldstein TA, et al. Myocardial blood volume is associated with myocardial oxygen consumption: an experimental study with cardiac magnetic resonance in a canine model. JACC Cardiovasc Imaging 2009;2:1313-20.

9. McCommis KS, Goldstein TA, Abendschein DR, et al. Roles of myocardial blood volume and flow in coronary artery disease: an experimental MRI study at rest and 
during hyperemia. Eur Radiol 2010;20:2005-12.

10. Wacker CM, Bauer WR. Myocardial microcirculation in humans--new approaches using MRI. Herz 2003;28:74-81.

11. Mahmod M, Piechnik SK, Levelt E, et al. Adenosine stress native T1 mapping in severe aortic stenosis: evidence for a role of the intravascular compartment on myocardial T1 values. J Cardiovasc Magn Reson 2014;16:92.

12. Levelt E, Piechnik SK, Liu A, et al. Adenosine stress CMR T1-mapping detects early microvascular dysfunction in patients with type 2 diabetes mellitus without obstructive coronary artery disease. J Cardiovasc Magn Reson 2017;19:81.

13. Liu A, Wijesurendra RS, Liu JM, et al. Gadolinium-Free Cardiac MR Stress T1-Mapping to Distinguish Epicardial From Microvascular Coronary Disease. J Am Coll Cardiol 2018;71:957-68.

14. Shah R, Sree Raman K, Walls A, et al. Gadolinium-Free Cardiovascular Magnetic Resonance Stress T1 Mapping in Patients With Chronic Kidney Disease. JACC Cardiovasc Imaging 2019;12:2083-5.

15. Jespersen L, Hvelplund A, Abildstrøm SZ, et al. Stable angina pectoris with no obstructive coronary artery disease is associated with increased risks of major adverse cardiovascular events. Eur Heart J 2012;33:734-44.

16. Petersen JW, Pepine CJ. Microvascular coronary dysfunction and ischemic heart disease: where are we in 2014? Trends Cardiovasc Med 2015;25:98-103.

17. Karamitsos TD, Leccisotti L, Arnold JR, et al. Relationship between regional myocardial oxygenation and perfusion in patients with coronary artery disease: insights from cardiovascular magnetic resonance and positron emission tomography. Circ Cardiovasc Imaging 2010;3:32-40.

18. Cerqueira MD, Weissman NJ, Dilsizian V, et al. Standardized myocardial segmentation and nomenclature for tomographic imaging of the heart. A statement for healthcare professionals from the Cardiac Imaging Committee of the Council on Clinical Cardiology of the American Heart Association. Circulation 2002;105:539-42.

19. Sharma P, Socolow J, Patel S, et al. Effect of Gd-DTPA$\mathrm{BMA}$ on blood and myocardial $\mathrm{T} 1$ at $1.5 \mathrm{~T}$ and $3 \mathrm{~T}$ in humans. J Magn Reson Imaging 2006;23:323-30.

20. Gan C, Lankhaar JW, Marcus JT, et al. Impaired left ventricular filling due to right-to-left ventricular interaction in patients with pulmonary arterial hypertension. Am J Physiol Heart Circ Physiol 2006;290:H1528-33.

21. Marcus JT, Vonk Noordegraaf A, Roeleveld RJ, et al. Impaired Left Ventricular Filling Due to Right
Ventricular Pressure Overload in Primary Pulmonary Hypertension: Noninvasive Monitoring Using MRI. Chest 2001;119:1761-5.

22. Marcus JT, Gan CT, Zwanenburg JJ, et al. Interventricular mechanical asynchrony in pulmonary arterial hypertension: left-to-right delay in peak shortening is related to right ventricular overload and left ventricular underfilling. J Am Coll Cardiol 2008;51:750-7.

23. Hardegree EL, Sachdev A, Fenstad ER, et al. Impaired left ventricular mechanics in pulmonary arterial hypertension: identification of a cohort at high risk. Circ Heart Fail 2013;6:748-55.

24. Tonelli AR, Plana JC, Heresi GA, et al. Prevalence and Prognostic Value of Left Ventricular Diastolic Dysfunction in Idiopathic and Heritable Pulmonary Arterial Hypertension. Chest 2012;141:1457-65.

25. Hardziyenka M, Campian ME, Reesink HJ, et al. Right ventricular failure following chronic pressure overload is associated with reduction in left ventricular mass: evidence for atrophic remodeling. J Am Coll Cardiol 2011;57:921-8.

26. Ryan JJ, Archer SL. Emerging concepts in the molecular basis of pulmonary arterial hypertension: part I: metabolic plasticity and mitochondrial dynamics in the pulmonary circulation and right ventricle in pulmonary arterial hypertension. Circulation 2015;131:1691-702.

27. Nitenberg A, Foult JM, Kahan A, et al. Reduced coronary flow and resistance reserve in primary scleroderma myocardial disease. Am Heart J 1986;112:309-15.

28. Kahan A, Allanore Y. Primary myocardial involvement in systemic sclerosis. Rheumatology (Oxford) 2006;45 Suppl 4:iv14-7.

29. Galiè N, Saia F, Palazzini M, et al. Left Main Coronary Artery Compression in Patients With Pulmonary Arterial Hypertension and Angina. J Am Coll Cardiol 2017;69:2808-17.

30. Meloche J, Lampron MC, Nadeau V, et al. Implication of Inflammation and Epigenetic Readers in Coronary Artery Remodeling in Patients With Pulmonary Arterial Hypertension. Arterioscler Thromb Vasc Biol 2017;37:1513-23.

31. Raman B, Ariga R, Mahmod M, et al. 011 Adenosine stress T1 mapping: a novel contrast free method to assess myocardial perfusion and ischaemia in hypertrophic cardiomyopathy. Heart 2017;103:A8.

32. Tsoumani Z, Miller C, Schmitt M, et al. 28Adenosine stress native T1 mapping demonstrates impaired myocardial perfusion reserve in non-ischemic dilated cardiomyopathy. Available online: https://academic.oup.com/ehjcimaging/ 
article/20/Supplement_2/jez111.006/5510818

33. Messroghli DR, Moon JC, Ferreira VM, et al. Clinical recommendations for cardiovascular magnetic resonance mapping of T1, T2, T2* and extracellular volume: A consensus statement by the Society for Cardiovascular Magnetic Resonance (SCMR) endorsed by the European Association for Cardiovascular Imaging (EACVI). J Cardiovasc Magn Reson 2017;19:75.

34. Vogel-Claussen J, Skrok J, Shehata ML, et al. Right and Left Ventricular Myocardial Perfusion Reserves Correlate with Right Ventricular Function and Pulmonary Hemodynamics in Patients with Pulmonary Arterial Hypertension. Radiology 2011;258:119-27.

35. Saunders LC, Johns CS, Stewart NJ, et al. Diagnostic and prognostic significance of cardiovascular magnetic resonance native myocardial T1 mapping in patients with pulmonary hypertension. J Cardiovasc Magn Reson 2018;20:78.

36. McCann GP, Gan CT, Beek AM, et al. Extent of MRI Delayed Enhancement of Myocardial Mass Is Related to Right Ventricular Dysfunction in Pulmonary Artery Hypertension. AJR Am J Roentgenol 2007;188:349-55.

37. Manders E, Rain S, Bogaard HJ, et al. The striated muscles in pulmonary arterial hypertension: adaptations beyond the right ventricle. Eur Respir J 2015;46:832-42.

38. Homsi R, Luetkens JA, Skowasch D, et al. Left Ventricular Myocardial Fibrosis, Atrophy, and Impaired Contractility in Patients With Pulmonary Arterial Hypertension and a Preserved Left Ventricular Function: A Cardiac Magnetic Resonance Study. J Thorac Imaging 2017;32:36-42.

39. Spruijt OA, Vissers L, Bogaard HJ, et al. Increased native T1-values at the interventricular insertion regions in precapillary pulmonary hypertension. Int J Cardiovasc Imaging 2016;32:451-9.

40. Arnold JR, Karamitsos TD, Bhamra-Ariza P, et al. Myocardial oxygenation in coronary artery disease: insights from blood oxygen level-dependent magnetic resonance imaging at 3 tesla. J Am Coll Cardiol 2012;59:1954-64.

41. Dass S, Holloway CJ, Cochlin LE, et al. No Evidence of Myocardial Oxygen Deprivation in Nonischemic Heart Failure. Circ Heart Fail 2015;8:1088-93.

42. Karamitsos TD, Dass S, Suttie J, et al. Blunted myocardial oxygenation response during vasodilator stress in patients with hypertrophic cardiomyopathy. J Am Coll Cardiol 2013;61:1169-76.

43. Grover S, Lloyd R, Perry R, et al. Assessment of myocardial oxygenation, strain, and diastology in
MYBPC3-related hypertrophic cardiomyopathy: a cardiovascular magnetic resonance and echocardiography study. Eur Heart J Cardiovasc Imaging 2019;20:932-8.

44. Dharmakumar R, Arumana JM, Tang R, et al. Assessment of regional myocardial oxygenation changes in the presence of coronary artery stenosis with balanced SSFP imaging at 3.0 $\mathrm{T}$ : theory and experimental evaluation in canines. J Magn Reson Imaging 2008;27:1037-45.

45. Beache GM, Herzka DA, Boxerman JL, et al. Attenuated myocardial vasodilator response in patients with hypertensive hypertrophy revealed by oxygenationdependent magnetic resonance imaging. Circulation 2001;104:1214-7.

46. Levelt E, Rodgers CT, Clarke WT, et al. Cardiac energetics, oxygenation, and perfusion during increased workload in patients with type 2 diabetes mellitus. Eur Heart J 2016;37:3461-9.

47. Shah R, Parnham S, Liang Z, et al. Prognostic Utility of Oxygen-Sensitive Cardiac Magnetic Resonance Imaging in Diabetic and Nondiabetic Chronic Kidney Disease Patients With No Known Coronary Artery Disease. JACC Cardiovasc Imaging 2019;12:1107-9.

48. Karamitsos TD, Arnold JR, Pegg TJ, et al. Patients with syndrome $\mathrm{X}$ have normal transmural myocardial perfusion and oxygenation: a 3-T cardiovascular magnetic resonance imaging study. Circ Cardiovasc Imaging 2012;5:194-200.

49. Ong P, Camici PG, Beltrame JF, et al. International standardization of diagnostic criteria for microvascular angina. Int J Cardiol 2018;250:16-20.

50. Dumitrescu D, Sitbon O, Weatherald J, et al. Exertional dyspnoea in pulmonary arterial hypertension. Eur Respir Rev 2017;26:170039.

51. Sun XG, Hansen JE, Oudiz RJ, et al. Exercise pathophysiology in patients with primary pulmonary hypertension. Circulation 2001;104:429-35.

52. Sitbon O, Gomberg-Maitland M, Granton J, et al. Clinical trial design and new therapies for pulmonary arterial hypertension. Eur Respir J 2019;53:1801908.

Cite this article as: Sree Raman K, Shah R, Stokes M, Walls A, Woodman RJ, Ananthakrishna R, Walker JG, Proudman S, Steele PM, De Pasquale CG, Celermajer DS, Selvanayagam JB. Left ventricular ischemia in pre-capillary pulmonary hypertension: a cardiovascular magnetic resonance study. Cardiovasc Diagn Ther 2020;10(5):1280-1292. doi: 10.21037/ cdt-20-698 